\title{
A Disembarking Notification Application for Public Transportation Using Inaudible Frequency
}

\author{
Myoungbeom Chung (iD) \\ College of Paideia, Sungkyul University, Anyang City 14097, Republic of Korea \\ Correspondence should be addressed to Myoungbeom Chung; nzine@sungkyul.ac.kr
}

Received 26 July 2021; Accepted 31 December 2021; Published 13 January 2022

Academic Editor: Ding Xu

Copyright (c) 2022 Myoungbeom Chung. This is an open access article distributed under the Creative Commons Attribution License, which permits unrestricted use, distribution, and reproduction in any medium, provided the original work is properly cited.

\begin{abstract}
Currently, many people enjoy videos and music content through their smart devices while using public transportation. However, because passengers focus so much on content on their smart devices, they sometimes forget to disembark and miss their destination stations. Therefore, in this paper, we propose an application that can notify users via smart devices when they approach the drop-off point in public transportation using an inaudible high frequency. Inaudible frequency signals are generated with announcements from speakers installed on subways and city buses. Smart devices receive and analyze the signals through their built-in microphones and notify users when they reach the drop-off point. We tested destination notifications with the proposed system and 10 smart devices to evaluate its performance. According to the test results, the proposed system showed $99.4 \%$ accuracy on subways and $99.2 \%$ accuracy on city buses. Moreover, we compared these results to those using only subway app in subways, and our proposed system achieved far better outcomes. Thus, the proposed system could be a useful technology for notifying smart device users when to get off public transport, and it will become an innovative technology for global public transportation by informing users of their desired stations using speakers.
\end{abstract}

\section{Introduction}

As the supply of smart devices has increased rapidly recently and the network speed has greatly improved due to $5 \mathrm{G}$ communication replacing $4 \mathrm{G}$ long-term evolution (LTE) communication, many people enjoy movies and music content using smart devices in various places. When people travel to work or school by public transportation, many people often use smart devices to watch videos, such as Internet lectures, YouTube, Netflix, and Watcha, and to listen to music with earphones. However, when people focus on enjoying the content of smart devices while traveling on public transportation, they often pass through the station where they have to get off. As a result, services to inform people of their stops in subways and buses were gradually required, and smart-device-based applications and service systems using various technologies have been provided to meet these demands.
Disembarking notification services are a technology that uses only the GPS of smart devices $[1,2]$, the Wi-Fi fingerprint based on Wi-Fi APs (Access Points) [3], and "bus stop Open API," which is public data substituted for the GPS of smart devices [4]. In the case of GPS-only technology, it is effective to use on buses, but because GPS signals are weak on subways, it is impossible to find the exact location. Wi-Fi fingerprint technology stores collected predata on servers based on the signal strength of the Wi-Fi AP located in each location, which can provide that information to the smart devices of users. Because this technology promises high accuracy in indoor spaces, it is possible to provide more effective information on subways. However, the user's smart device needs to communicate with the information provider server to receive the information after recognizing the Wi-Fi signal, and if various facilities or devices are installed or missing around the Wi-Fi AP, it is necessary to store a new Wi-Fi fingerprint. Furthermore, this technology is not 
available for iOS due to security issues surrounding the use of APs installed on subways, so it is only available on Android [5]. Technology that utilizes public data integrates GPS information for each bus operated by a bus company on a server and sends the data to a smart device to inform the user of the current location and notify them of the drop-off. However, the technology can only inform users if they need current location information, and the disadvantage is that continuous communication with the server is required to notify users when public transportation reaches the drop-off point.

Therefore, in this paper, we propose a new public transportation disembarkation notification service using smart devices and inaudible high frequencies that can be output from speakers installed in subways and buses. A microphone built into a smart device can recognize sound signals ranging from $20 \mathrm{~Hz}$ to $22 \mathrm{kHz}$, a range of hearing frequencies that people can normally hear, but the application can also analyze received sounds to detect specific high-frequency signals that are not heard by people [6]. At this time, the specific signals are generated with station notification broadcasting using speakers installed in public transportation. They consist of two high frequencies ranging from $18 \mathrm{kHz}$ to $20 \mathrm{kHz}$, which have been used in existing smart guidance applications and data communication studies. Therefore, people in the space where the signal occurs do not recognize the specific signal. The user's smart devices then analyze the received sound and provide the user with a drop-off notification when the drop-off point and the signal detection value match if the specified signal is detected in the announcement. Since there are so many public bus and subway lines in each city, data matching the received high-frequency signal with the subway and bus stops are received only once through the server when the user enters the relevant subway line or bus number into the application. High-frequency pairs are used to match each stop station on that bus or subway. To verify the performance of the proposed system, we built a smart-device-based public transportation disembarking notification application and service server and tested drop-off notification experiments using a total of 10 smart devices. For the experiment comparing subway disembarking notifications, we tested the disembarking notifications 1,000 times using the proposed application and the disembarking notification function of the Kakao subway. Moreover, for the experiment of comparing disembarking notifications on city buses, we developed an application for disembarking notifications using Open API information and tested the disembarking notification 1,000 times using the proposed application and the Open API application. The experiments show that the proposed application has $99.4 \%$ accuracy on subways and $99.2 \%$ accuracy on city buses. Furthermore, comparative experiments show that the proposed method has higher accuracy than Open API for city buses and Kakao subway disembarking notifications, and we also confirmed that the proposed method is superior in battery consumption. Thus, the proposed application and system will be useful for passengers using public transportation, as they use the microphones of smart devices and inaudible high frequencies to convey disembarking notifications to users. Indeed, it will be an excellent technology that can be commercialized in various public transportation services around the world to guide travelers to their next destination using built-in speakers on subways in such cities as the following: Hong Kong; Lisbon, Portugal; Tokyo and Osaka, Japan; Bangkok, Thailand; Seoul and Busan, South Korea; and New York, USA.

The composition of this paper is as follows. Section 2 describes existing studies on disembarking notification services in public transportation and existing studies using inaudible high frequency in the audible frequency domain. Section 3 describes the flow of inaudible frequency signal processing techniques and service systems used in proposed applications and servers. Section 4 presents experiments and results for verifying the performance of public transportation disembarking notification services using the proposed system and comparative experiments and results with existing applications, and Section 5 presents conclusions and future research directions.

\section{Previous Work}

In this section, we review existing studies on disembarking notification services in public transportation and existing studies using inaudible high frequency in the audible frequency range used in this study. Early public transportation services only provided predictive information that users would arrive at a station at a fixed time for the service hours of subways and buses. As smart devices began to spread rapidly, public transportation service applications based on smart devices, such as SCOTTY in Austria [7], NextStop [8], and TransitTimes+ [9] in the United States, provided operational information. There are also increasingly active services that provide alarms and notifications to users as they approach set-up points or locations. In Malaysia, bus notifications using GPS information from user smart devices and geofence [10] and real-time bus location tracking applications using RFID have been proposed [11]. In Korea, a public transportation disembarking notification service was developed and provided in 2012 with Seoul city bus and subway disembarking notification applications using operating time information and GPS information on city buses. In addition, TMap, KakaoMap, Seoul Bus Information, and Smart Subway have recently provided public transportation disembarking notification services by collecting GPS information and Wi-Fi fingerprint information on servers and delivering it to smart devices. However, most of these services are limited: only when users' smart devices are continuously receiving bus and subway information from the server using communication pathways such as LTE, Wi-Fi, or GPS with Wi-Fi fingerprint information. In other words, notification services can often malfunction if they do not receive operational information via LTE or Wi-Fi, GPS information is inaccurate, or Wi-Fi fingerprint information changes. Even on buses, public transportation disembarking notification services based on GPS information initially used only GPS location values built into smart devices. However, due to inaccurate GPS values in the vehicle, recent 
disembarking notification services have utilized bus GPS information integration servers, which are public data. The recently proposed smart bus system using BLE beacons and computer vision also uses Open API information to implement drop-off notifications, drop-off bell automatic operation, and so on [12].

The existing studies using high frequencies use $18 \mathrm{kHz}$ to $22 \mathrm{kHz}$, an inaudible frequency region in the audible frequency range $(20 \mathrm{~Hz}$ to $22 \mathrm{kHz})$, to transmit or control specified signals using built-in speakers and microphones of smart devices. Bihler uses $20 \mathrm{kHz}$ and $22 \mathrm{kHz}$ high frequencies to transmit signals with frequency-shift keying (FSK) technology and applies Hamming code to correct errors in the transmission signal. He proposed a smart guide system that sends the trigger signal to a smart device to provide information [13]. Chung proposed a data-sharing method between smart devices using Wi-Fi and high-frequency signals that are composed of two base signals and one variable signal to transmit signals more strongly [14]. He then proposed an application that could deliver high-frequency signals to TV viewers to provide advertisements for certain products [15]. The method uses three high frequencies as signal values for data transmission, which are aimed at accurately sending a specific signal to a smart device rather than sending a lot of data using high frequencies. Chung also proposed a high-frequency near-field communication method for data communication between PCs and smart devices using only high-frequency signals, and he applied cyclic redundancy check (CRC) to increase their accuracy [16]. Thus, the existing methods collect surrounding sound information where smart devices are located through built-in microphones, detect certain highfrequency signals from the collected sounds, and analyze whether the signals are signals for data transfer. In addition, smart devices perform services such as notifying users and forwarding information from values that match the transmitted signal.

\section{Disembarking Notification Application for Public Transportation Using Inaudible Frequency}

In this section, we explain the proposed application and service systems for public transportation disembarking notifications using smart devices and inaudible frequencies. The overall flow of the proposed system is shown in Figure 1.

In Figure 1, the user enters the bus number or subway line before or after getting on public transportation using the proposed application. The smart device sends the number and transportation type to the server (1) and receives bus station data and subway line data from the server (2). The smart device then displays the current location of the subway line or bus route information, and the user sets the destination at which they want to get off (3). The proposed application collects surrounding sounds, announcements, and high-frequency sounds from the smart device's built-in microphones (4), and public transport delivers announcements of each station through speakers while simultaneously sending out specific high-frequency signals embedded in the announcements for each station (5). The proposed application analyzes whether more than one high frequency occurs by conducting real-time fast Fourier transform (FFT) processing of all collected sounds (6) and compares the values of a user-specified drop-off destination and a station schedule to stop when a pair of high frequencies is detected (7). That is, if the two values match, the application will finally notify the user of the drop-off site (8).

The user's bus number or subway line input and transmission communicate with the server using $\mathrm{Wi}-\mathrm{Fi}$ and LTE together with GPS data to determine the user's current location, as shown in Figure 2 (1).

The server sends the name of the stations, the signal value for each station, and the name of the bus station closest to the user's GPS information to the smart device in the case of bus routes, as shown in Figure $2(2,3)$. For example, if a user enters Incheon airport bus "6017" and sends the bus number to the server, the server sends the bus's route information to a smart device, as in Figure 3.

The total number of bus stations in Figure 3 is 16, and two pairs of high-frequency signals for each station are selected in units of $100 \mathrm{kHz}$ over the $18 \mathrm{kHz}$ to $20 \mathrm{kHz}$ range. If only one high-frequency signal were used, it is possible that the same high frequency would be generated by the surrounding noises; therefore, we used the detection of two high frequencies, not one, generated simultaneously as a signal. However, if a pair of high frequencies is within $600 \mathrm{~Hz}$, the frequency intervals differ by more than $600 \mathrm{~Hz}$ because errors can occur due to frequency interference [17]. In other words, a pair of high frequencies matching a station can consist of 105 pairs, including " $18.0 \mathrm{kHz}$ and $18.7 \mathrm{kHz}$," " $18.0 \mathrm{kHz}$ and $18.8 \mathrm{kHz}, " .$. " $19.3 \mathrm{kHz}$ and $20.0 \mathrm{kHz}$," and each stop in Figure 3 can be matched with a pair of highfrequency signals, as shown in Table 1.

In Table 1, a pair of high-frequency data consisting of $18.0 \mathrm{kHz}$ and $18.7 \mathrm{kHz}$ are recognized as 00 values, while data consisting of $18.0 \mathrm{kHz}$ and $20.2 \mathrm{kHz}$ are recognized as hexadecimal $0 \mathrm{~F}$ values. In other words, we can see that the 00 value is set as the "Seoul National Univ." terminal station (station number 01), $0 \mathrm{E}$ as Incheon International Airport Terminal 1 (station number 15), and $0 \mathrm{~F}$ as Incheon International Airport Terminal 2 (station number 16). This highfrequency signal produces a broadcast file so that it can occur simultaneously when announcing a stop through speakers on buses and subways. At this time, inaudible frequency signals do not occur throughout the announcement, and they occur twice for one second at a time interval for a total of three seconds. When a pair of high frequencies is detected twice rather than once, the designated signal is considered to have occurred. This is because, as Chung's experimental results showed, high-frequency detection accuracy in smart devices is improved when generating a high frequency for more than 2.8 seconds. Figure 4 represents the time and frequency values of the signals sent when a stop station is announced.

In Figure 4, the pair of high-frequency signals are $18.0 \mathrm{kHz}$ and $18.7 \mathrm{kHz}$, which occur for three seconds (n) and twice (k). The signals occur from 0.5 seconds to 3.5 


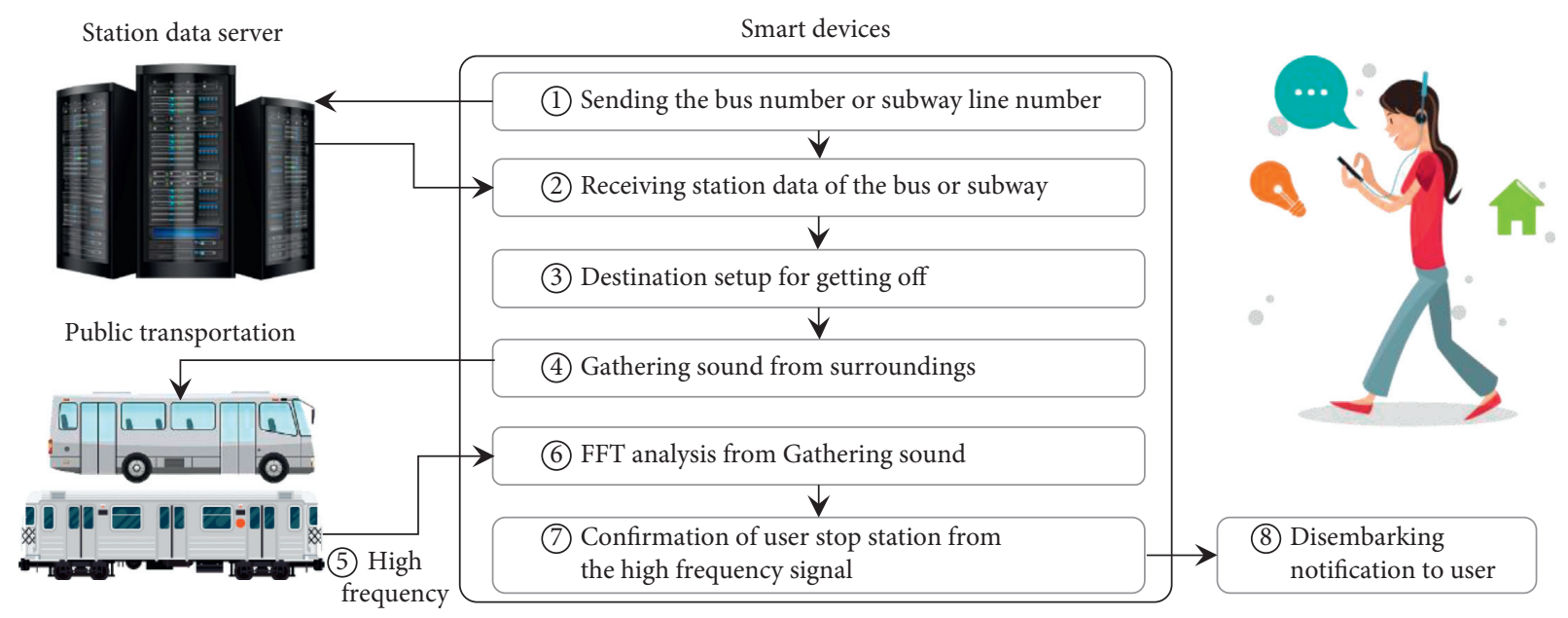

Figure 1: The flow of the disembarking notification system using smart devices and inaudible frequency.

seconds at $18.0 \mathrm{kHz}$ (red solid line below) and $18.7 \mathrm{kHz}$ (blue dotted line above); they occur for 1 second in a 3 second period, pause for 1 second, and then occur again for 1 second. In other words, it generates the same signal once from the first 0.5 to 1.5 seconds and once again from 2.5 to 3.5 seconds (twice in total). As this pair of high frequencies occurs through speakers with an announcement, smart devices located in public transportation continuously analyze whether inaudible frequencies above $18.0 \mathrm{kHz}$ exist around them through built-in microphones. In addition, when a high frequency is detected, the smart device performs one repeat check to verify that it represents the same value as the initial detected high-frequency pair. In Figure 5, the pseudocode is an expression of the processing for detecting a pair of inaudible frequencies on a smart device.

In Figure 5, $\mathbf{A}_{\mathbf{t}}$ is the audio value collected from the builtin microphone of a smart device at time $\boldsymbol{t}$, and $\mathrm{F}_{\mathbf{t}}$ is the bin value for each frequency obtained by converting $\mathbf{A}_{\mathbf{t}}$ to FFT. Thus, the high frequencies are sorted in order with high bin values (sortByBin), and if both the first high frequency with the most bin and the second-highest frequency with the second most bin exceed $18.0 \mathrm{kHz}$, they are entered in variables $\mathbf{a}$ and $\mathbf{b}$, respectively, and wait a certain amount of time ( $\mathbf{m}$ seconds) for detection. After waiting, the smart device collects ambient audio values and calculates the $\mathbf{F}_{\mathbf{t + 1}}$ values through FFT conversion to align them. When both the first and second high frequencies exceed $18.0 \mathrm{kHz}$, the existing a frequency values and the first high-frequency values are compared, and the existing $\mathbf{b}$ frequency values and the second high-frequency values are compared. If they all match, the smart device uses both values $\mathbf{a}$ and $\mathbf{b}$ to fetch station information and then notify the user of the drop-off.

Finally, if a station changes in the future, the guiding sound of the changed station must be recorded anew. At this time, we can also add the specific high-frequency signal used in the proposed method to the guide broadcasting sound. The proposed method can consist of up to 105 pairs with the high-frequency values of each station, as shown in Table 1 , and it is used after receiving each station's information and signal values from the route server when the user inputs the bus number or subway line information. Therefore, if the stop station changes in the future, the proposed application can easily change the station information and signal value on the route server and add the corresponding high frequency to the newly recorded broadcasting sound.

\section{Experiments and Evaluations}

This section explains the disembarking notification application using smart devices based on the inaudible frequency and analyzes user disembarking notification experiments and their results using the proposed application and server system. The screen configuration of the proposed application for disembarking notifications is shown in Figure 6.

Figure 6 shows a screen that appears when a user first runs the proposed application. After selecting which bus or subway the user is aboard, the user enters the bus number or subway line and presses the "Send" button to receive the route information from the server. The application will display the screen in Figure 7 when it receives a bus route from the server and the screen in Figure 8 when it receives a subway line.

In Figure 7, "Bus number: 6017" is the bus route number entered by the user followed by the values shown below:

(i) GPS: GPS location value of user

(ii) High frequencies: detected inaudible frequency values

(iii) Destination: user's specified destination

(iv) Current station: recent station from detected inaudible frequency values

In Figure 7, the current high-frequency values detected from ambient sounds are $18.0 \mathrm{kHz}$ and $18.9 \mathrm{kHz}$, shown in the graph on the left and "High Frequencies" on the right, which represent the third station on Bus Route 6017, "Bongcheon Sageori (03)," and a Bitdata value of 02.

In Figure 8, "Subway line: 2" is a subway line entered by the user, and the rest represents the same values as in Figure 7, except for GPS information. The current high- 


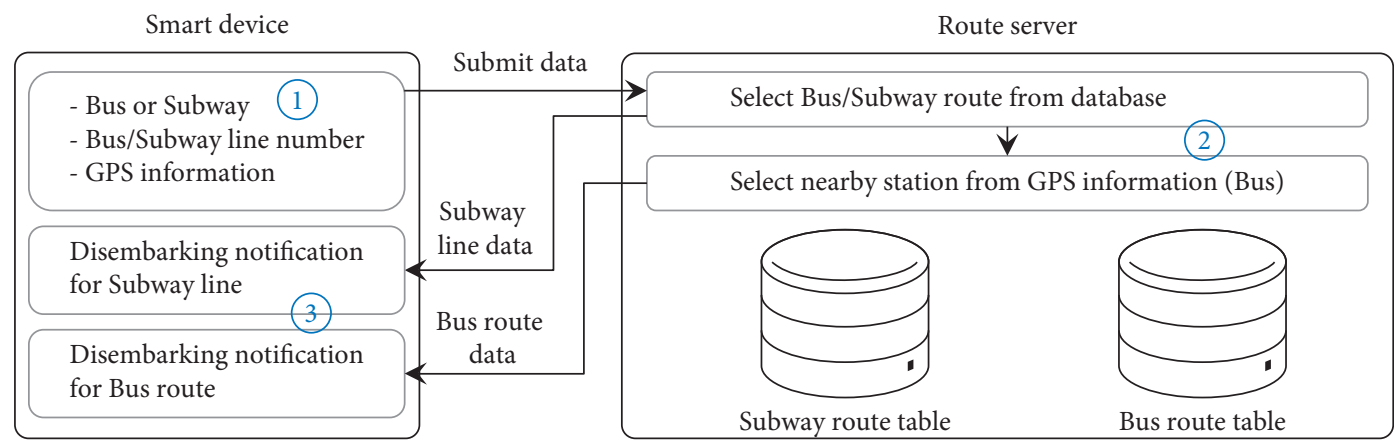

Figure 2: Data transmission for bus route or subway line data between the smart device and route server.

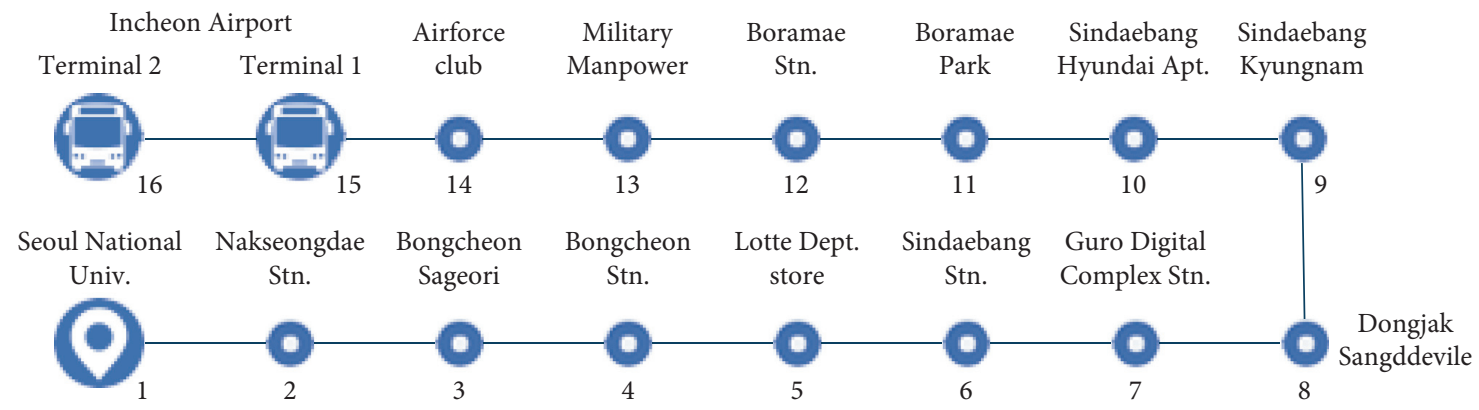

FIgURE 3: The 6017 bus (Seoul, South Korea) route received from the server system.

TABLE 1: Matching bus stations and a pair of high frequencies for each bit data.

\begin{tabular}{lccc}
\hline Bit data & Bus station & Inaudible frequencies & Station no. \\
\hline 00 & Seoul National Univ. & $18.0 \mathrm{kHz}$ and $18.7 \mathrm{kHz}$ & 01 \\
01 & Nakseongdae Stn. & $18.0 \mathrm{kHz}$ and $18.8 \mathrm{kHz}$ & 02 \\
02 & Bongcheon Sageori & $18.0 \mathrm{kHz}$ and $18.9 \mathrm{kHz}$ & 03 \\
03 & Bongcheon Stn. & $18.0 \mathrm{kHz}$ and $19.0 \mathrm{kHz}$ & $\ldots$. \\
$\ldots \ldots$ & $\ldots \ldots$ & $18.0 \mathrm{kHz}$ and $20.0 \mathrm{kHz}$ & 04 \\
$0 \mathrm{D}$ & Airforce Club & $18.1 \mathrm{kHz}$ and $18.8 \mathrm{kHz}$ & 14 \\
$0 \mathrm{E}$ & Incheon International Airport Terminal 1 & $18.1 \mathrm{kHz}$ and $18.9 \mathrm{kHz}$ & 15 \\
$0 \mathrm{~F}$ & Incheon International Airport Terminal 2 & 16 \\
\hline
\end{tabular}

frequency values detected from ambient sounds are $18.1 \mathrm{kHz}$ and $19.4 \mathrm{kHz}$, as shown in the graph on the left and "High Frequencies" on the right, corresponding to "City Hall (21)," the 21st station on Subway Line 2. The "Set" button, located next to "Destination," is used by the user to set up a station to get off the bus or subway. It displays a list of bus and subway stations at the touch of a button and allows the user to choose. When the user selects a drop-off station from the list, the station name appears under "Destination" in Figures 7 and 8 , and the proposed application sends a disembarking notification to the user when the inaudible frequency signal values match the destination values. The proposed application continuously proceeds with high-frequency collection in background mode, even if the user is using different content. When the destination value and the current destination station value are the same, the proposed application shows the disembarking notification as a local notification. The user reruns the proposed application by touching the disembarking notification, even if the user is using other content, and the proposed application informs the user that they are arriving at the destination, as in Figure 9. Figure 9 shows that the bus will be approaching the "Incheon International Airport Terminal 1 (15)," and the user can get off without skipping their destination by looking at the disembarking notification. If the user transfers, they will touch the "Confirm" button while riding the bus or subway. If the user then enters the bus number or subway line in the same way and selects the destination, the user can receive the next drop-off notification.

To verify the accuracy of the proposed application, we set 43 subway stations included in Line 2 of Seoul subway, South Korea, to values from 1 (Seocho) to 43 (Seoul Nat'l Univ. of Education) and matched each value with the subway station name, as in Figure 10.

The server environment for providing information on bus numbers and subway lines used Apache 2.2.14, MySQL 5.1.39, and PHP 5.2.12, and the hardware used was an Intel(R) Core (TM) i5 CPUs 750 with 8 G Ram. In Figure 10, the "Seocho" station has a value of 1 bit data (00), and the proposed application recognizes the "Seocho" station if it 


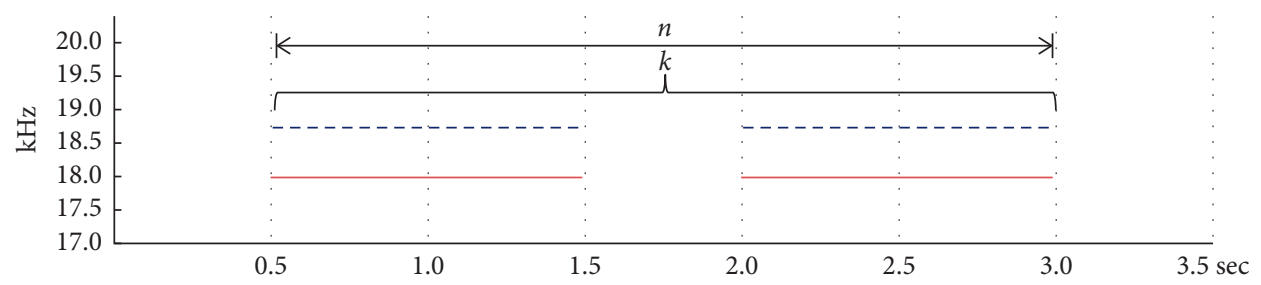

Figure 4: An example of a proposed pair of high frequencies for disembarking public transportation.

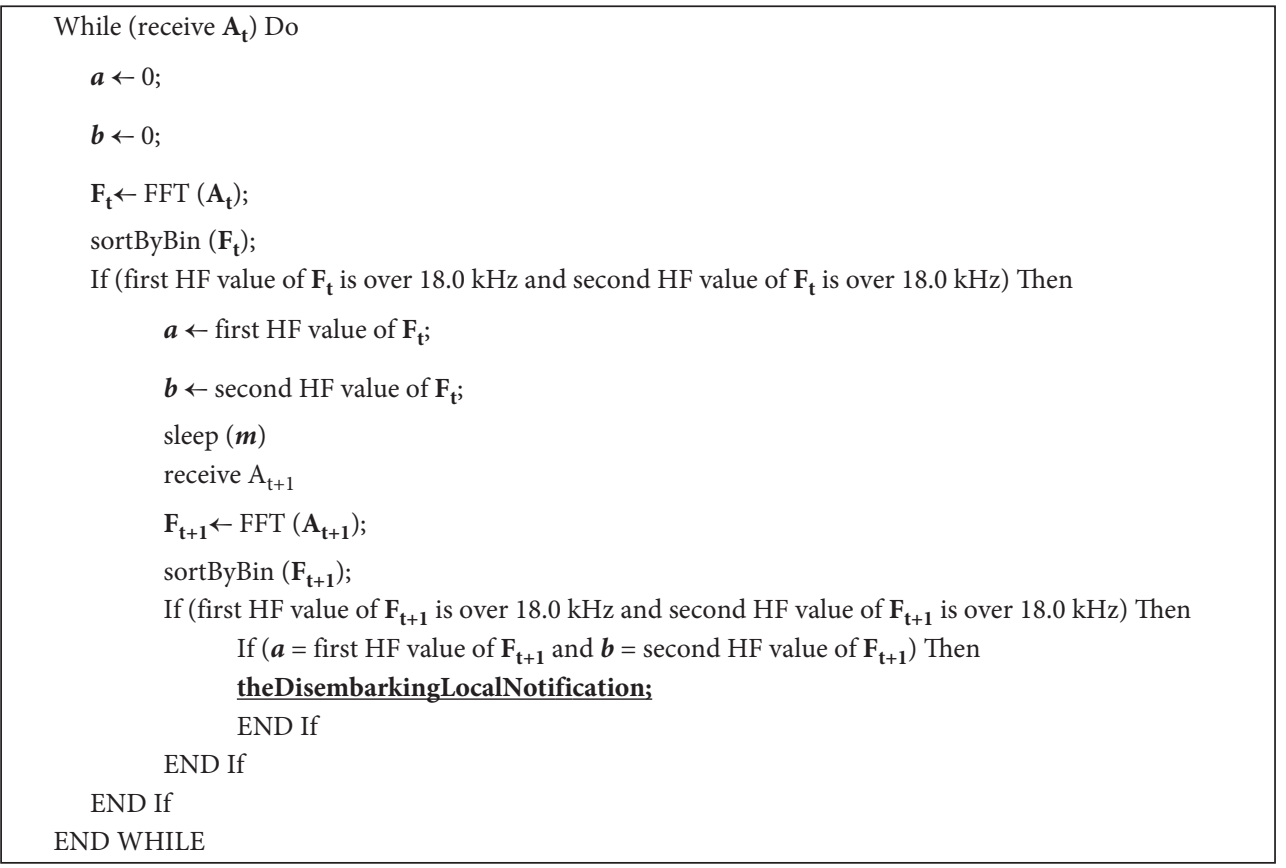

Figure 5: The pseudocode for high-frequency detection to notify users of disembarking locations.

recognizes a pair of high-frequency values in Table 1 $(18.0 \mathrm{kHz}$ and $18.7 \mathrm{kHz})$ from ambient sound data. The experiments in this paper did not set high-frequency values for "Kkachisan," "Sinjeongnegeori," "Yangcheon-gu Office," and "Dorimcheon" stations on the left and "Yongdap," "Shindap," "Yongdu," and "Shinseol-dong" stations on the right, which are not included in the cycle, as experiments must be conducted continuously during the line 2 cycle. We then stored subway line and bus station information for the experiments, as shown in Figure 11, on the server.

Figure 11(a) stores a part of the station information for Seoul subway line 2, indicating that both line_no are 2. s_name stores the names of each station, and s_no indicates each station's number. Figure 11(b) stores a part of bus station 6017's information, indicating that the bus_no is 6017. lati represents the latitude value, and longi represents the longitude value. Ten participants were recruited for the experiment, and each participant installed the proposed application on their own smart device. A total of 10 smart devices were used in the experiment: 2 each of iPhone 10, iPhone 11, iPhone 12, Galaxy S20, and Galaxy Note 20. The speakers that generated high-frequency signals at subway announcements were laptop speakers, and high-frequency generation was developed and carried out by JavaScript program-based web applications. In the experiment, all the participants were located in one compartment of subway line 2, and the distance between the participants' smart devices and laptops was maintained within 5 meters. In addition, each participant was required to set a disembarking destination at or before three stations from the departure station. Participants in the experiment checked that local notifications worked well before arriving at the destination they set up. We tested the disembarking notification a total of 100 times, and Figure 12 is a graph showing the results of smart devices recognizing the inaudible frequency and notifying users with drop-off alerts. In Figure 12, iX is an iPhone 10; i11 is an iPhone $11 ; \mathrm{i} 12$ is an iPhone 12; G20 is a Galaxy S20; and N20 is a Galaxy Note 20. The numbers shown on the graph for each device represent the number of times users were notified of the drop-off by matching the values of the station arriving with the user's destination. We can see that five smart devices performed the correct disembarking notification 100 times, four smart devices did so 99 times, and the third iPhone 11 did so 98 times. We expect that the reason why the iPhone 11 is less accurate than other devices is that it belonged to the participant who was located furthest away from the laptop and sometimes did not recognize the specified high frequency because it was more than 


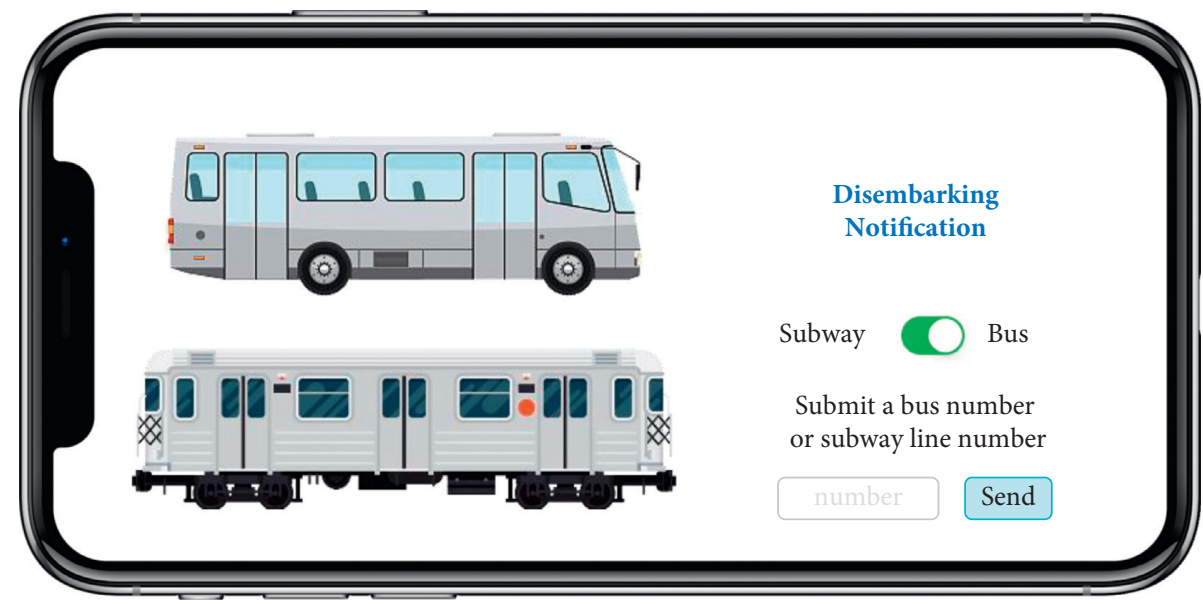

FIGURE 6: Screen composition of the proposed application for disembarking notifications.

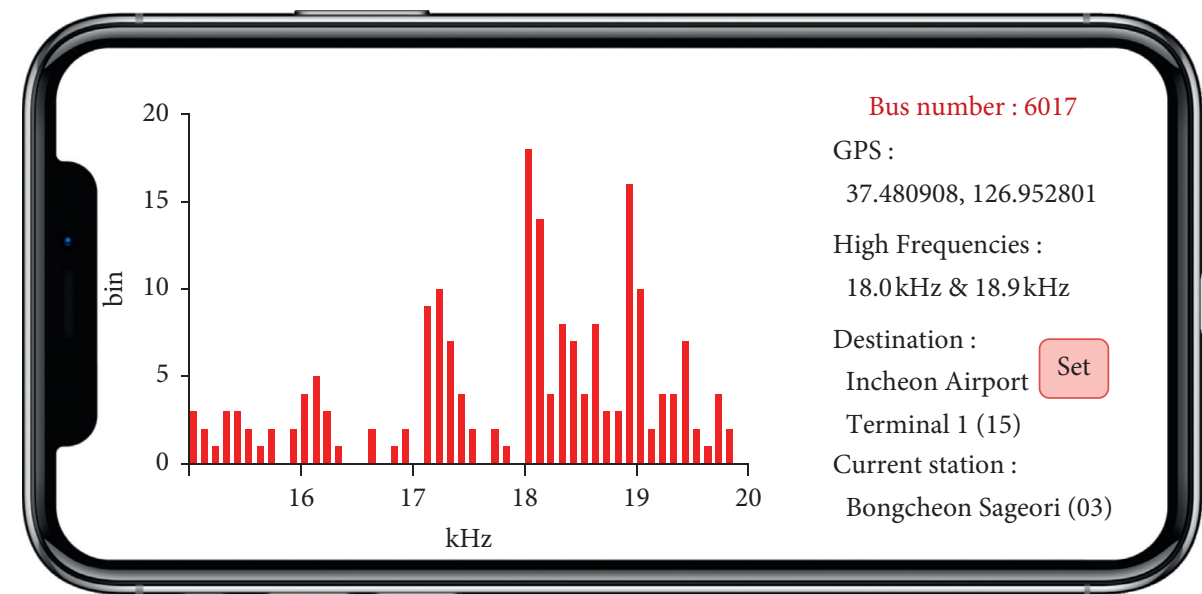

FIGURE 7: Screen composition of the proposed application for disembarking on public city buses.

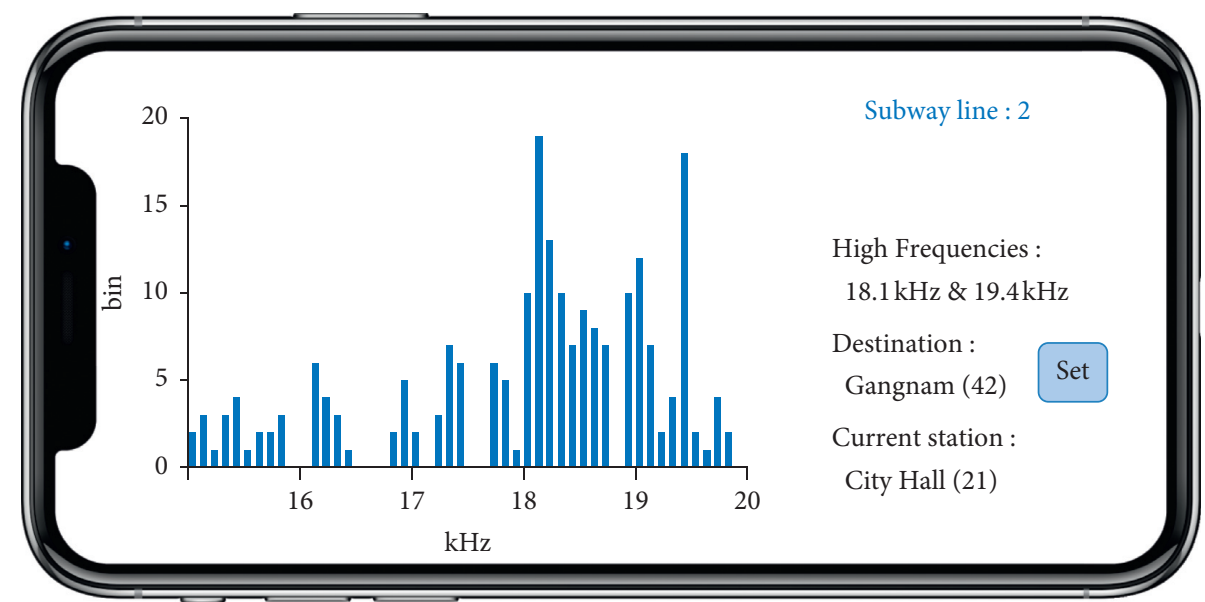

Figure 8: Screen composition of the proposed application for disembarking at subway line number 2.

five meters away from the laptop. Therefore, in this experiment, the proposed application showed $99.4 \%$ accuracy.

In the next experiment, the participants were divided into two teams composed of five participants, and the experiments were conducted in the same way in two different compartments of the subway, with the results shown in Figure 13.

In Figure 13, the first subway compartment (Space 1) was $99,100,100,100$, and 100, with $99.8 \%$ accuracy. The second subway compartment (Space 2) was 100, 99, 99, 100, and 98, 


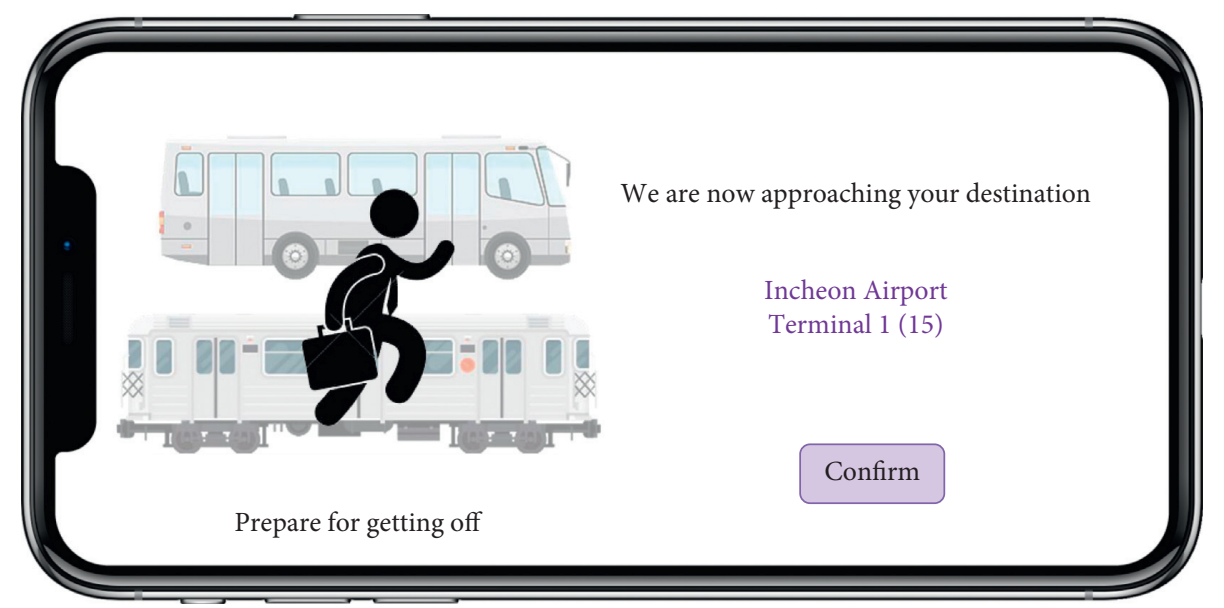

FIgURE 9: The screen of the application when the city bus or subway is approaching the destination station.

with 99.2\% accuracy. In other words, the second experiment showed that the disembarking notification accuracy was 99.5\% overall. We expect that the reason why the second subway car was less accurate than the first subway car was because there were more people in the second space and some passengers moved to get off when subway announcements and high-frequency signals were generated, interfering with high-frequency recognition on smart devices.

Since iOS is not available, the subsequent experiment consisted of eight participants using a total of eight Androidbased Galaxy S20s and Galaxy Note20s. Participants in the experiment were divided into two teams of four and took the same subway together. One team used the proposed application, and the other team used the Kakao subway dropoff notification application. The number of experiments was the same as in the previous experiment, and the results of the disembarking notification experiment are shown in Figure 14.

In Figure 14, the proposed method shows 99, 100, 100, and 98 accurate notifications, with an average accuracy of $99.2 \%$. We think that 99 on the G20 and 98 on the N20 did not operate the disembarking notifications because participants were either at the farthest distance from the speaker or because participants blocked the microphone of the smart device. On the other hand, the Kakao subway drop-off notification application shows 92, 91, 90, and 91 accurate notifications, with an average accuracy of $91 \%$. In most cases, we expect that the reason why $100 \%$ accuracy does not appear is that position estimation errors occur depending on the network environment and speed in the process of verifying AP information after the subway arrives at the station. Thus, we think that depending on the quality of the arriving station's network, the notification for getting off may be faster and later, but most of the errors are late.

In another experiment, we measured the battery consumption of each smart device together at the beginning of the comparison experiment above. Before the start of the experiment, the battery in each smart device started at $100 \%$ charge, with a total of 10 measurements taken when passing the initial start position. Experiments to measure battery consumption have refrained from using other applications during the experiment because there are significant differences in the execution of other applications, Wi-Fi, GPS, screen brightness control, and other sensors. The proposed application turned off Wi-Fi because it did not need to use Wi-Fi after bringing in subway line data, and the Kakao subway drop-off notification application turned it on during the experiment because Wi-Fi was required. Eight smart devices set the same screen brightness, and all sensors except Wi-Fi had the same values. Figure 15 compares the results of the battery consumption of the proposed method and the Kakao subway disembarking notification. As shown in Figure 15, the proposed application consumed $52 \%$ of the battery in 10 trials, and the Kakao subway disembarking notification application consumed $85.5 \%$. We know that the reason why Kakao subway disembarking notification applications consume more than $33.5 \%$ of battery power compared to the proposed application is that Wi-Fi and LTE are constantly used to get AP values as they approach each subway station for drop-off notification.

In the next experiment, we conducted a city bus disembarking notification experiment using a total of 10 smart devices, the same as in the previous subway experiment. There were once again 10 participants, and speakers and applications to transmit high-frequency signals were used similarly to the subway experiment. The experiment used the same city bus (6017) as in Figure 3, and participants were all on board at Seoul National University Station, the last stop of bus 6017. Participants then sat in a comfortable position on the bus while speakers and laptops generating highfrequency signals were placed in the central position of the bus. Participants set a destination setting of more than 3 stations at the departure station, and they checked whether the disembarking notification worked well when an announcement was made before arriving at the designated destination. For example, after a participant gets on at Seoul National University Station in Figure 3, the nearest stop designation can be set after Bongcheon Station. Participants will then check whether the disembarking notification works when the announcement is made at Bongcheon Station. In 


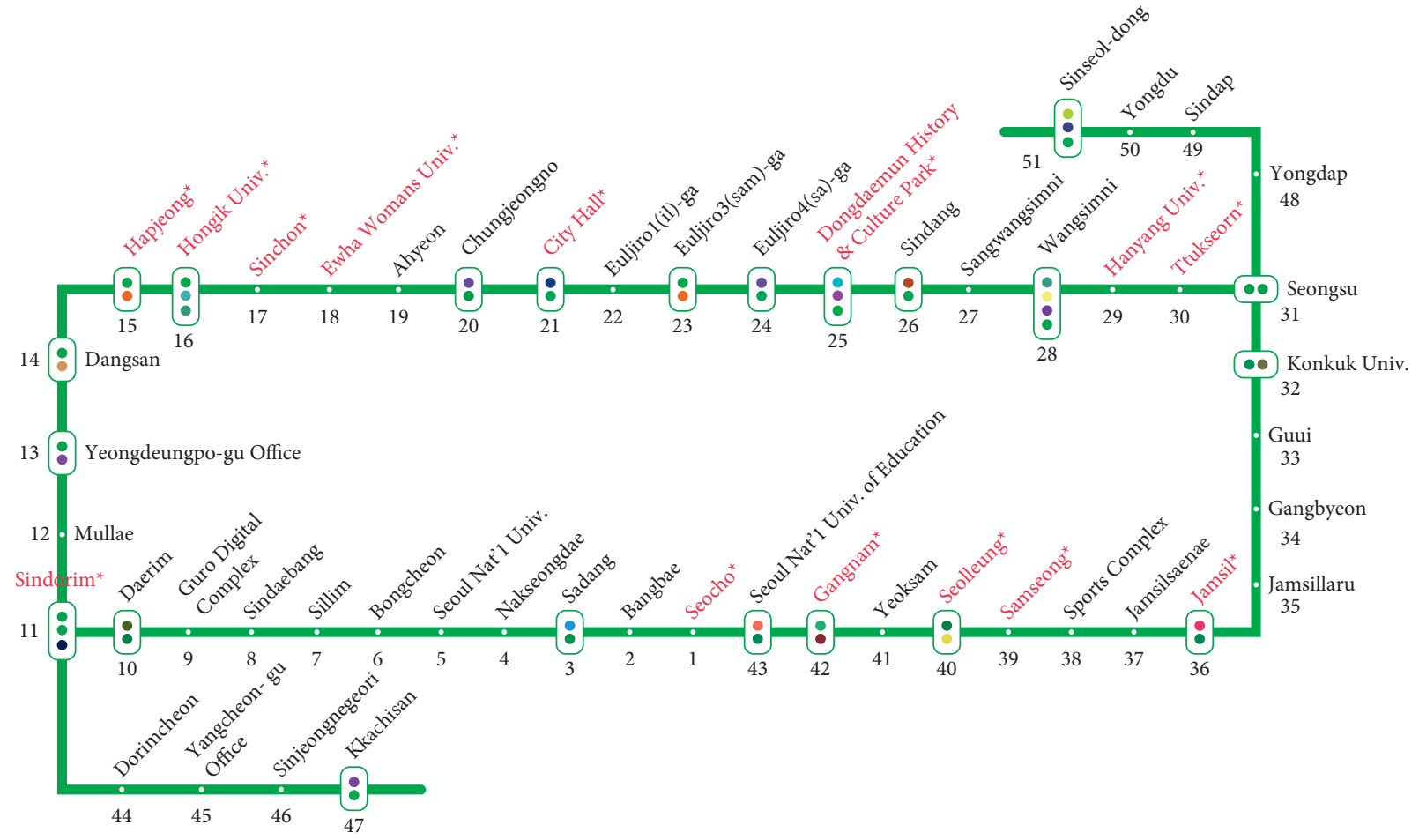

FIgURE 10: Seoul subway, South Korea, line 2 map and high-frequency values for each subway station.

\begin{tabular}{|l|c|c|c|}
\hline num & line_no & S_name & S_no \\
1 & 2 & Seocho & 1 \\
2 & 2 & Bangbae & 2 \\
3 & 2 & Sadang & 3 \\
4 & 2 & Nakseongdae & 4 \\
5 & 2 & Seoul Natl Univ. & 5 \\
6 & 2 & Bongcheon & 6 \\
7 & 2 & Sillim & 7 \\
8 & 2 & Sindaebang & 8 \\
9 & 2 & Guro Digital Complex & 9 \\
10 & 2 & Daerim & 10 \\
\hline
\end{tabular}

(a)

\begin{tabular}{|l|c|c|c|c|c|}
\hline num & bus_no & S_name & S_no & lati & longi \\
\hline 1 & 6017 & Seoul National Univ. & 1 & 37.45988 & 126.94971 \\
2 & 6017 & Nakseongdae Stn. & 2 & 37.46814 & 126.95797 \\
3 & 6017 & Bongcheon Sageori & 3 & 37.48123 & 126.95313 \\
4 & 6017 & Bongcheon Stn. & 4 & 37.48270 & 126.94211 \\
\hline 5 & 6017 & Lotte Dept. store & 5 & 37.48980 & 126.92530 \\
6 & 6017 & Sindaebang Stn. & 6 & 37.48778 & 126.91304 \\
\hline 7 & 6017 & Guro Digital Complex Stn. & 7 & 27.48551 & 126.90166 \\
8 & 6017 & Dongjak Sangddevile & 8 & 37.48881 & 126.90642 \\
\hline 9 & 6017 & Sindaebang Kyungnam & 9 & 37.49185 & 126.90893 \\
10 & 6017 & Sindaebang Hyundai Apt. & 10 & 37.49535 & 126.91166 \\
\hline
\end{tabular}

(b)

Figure 11: Station data example of subway line 2 and city bus 6017 for disembarking notification: (a) a part of the database table for Seoul subway, South Korea, line 2 and (b) a part of the database table for city bus 6017 .

addition, this participant can designate and confirm Guro Digital Complex Station, which is located after 3 stops, as designating the next stop. In other words, 1 participant can test 5 disembarking notifications from the starting station to the end station when riding a bus. Therefore, this experiment tested 2 round trips and 20 disembarking notifications per day and did so for 5 days to test it 100 times for each participant. Figure 16 is a result graph of participants' smart devices recognizing inaudible frequency signals and delivering disembarking notifications to users.
In Figure 16, we can see that 5 smart devices correctly delivered disembarking notifications 100 times (100\%), 3 smart devices did so 99 times, G20 did so 98 times, and N20 did so 97 times accurately. We think that the reason why the N20 is less accurate than other devices is that the participant sat farthest from the speaker generating the high frequency compared to the other participants, and therefore, the N20 did not always recognize the high frequency. Therefore, in this experiment, the proposed applications and server systems showed $99.2 \%$ disembarking notification accuracy. 


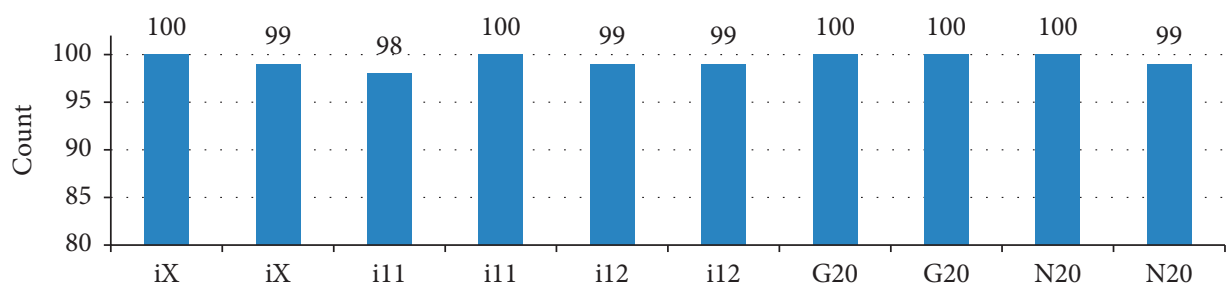

FIgURE 12: Disembarkation notification results on the subway of the paired high frequencies from each smart device.

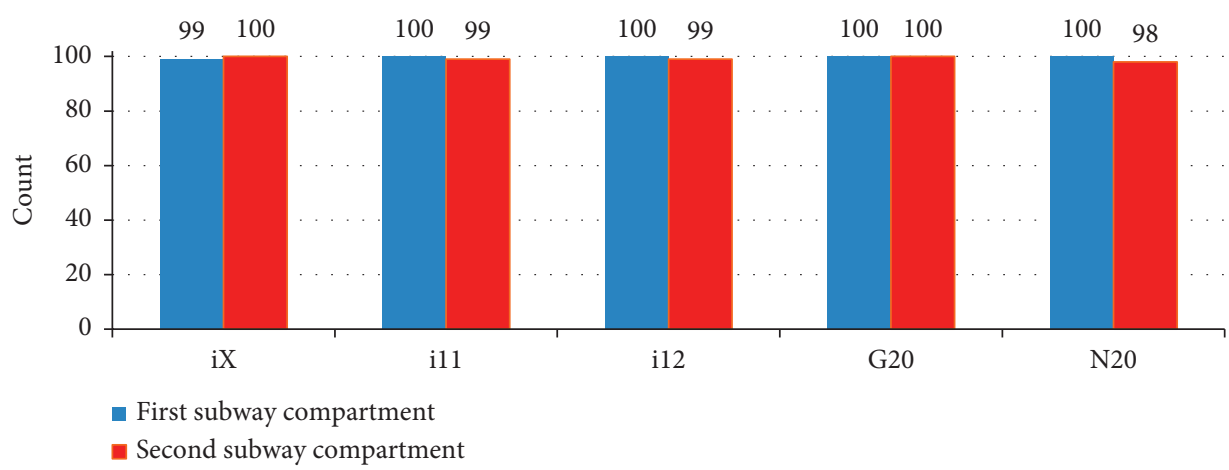

FIGURE 13: Disembarkation notification results in two different compartments of the subway.

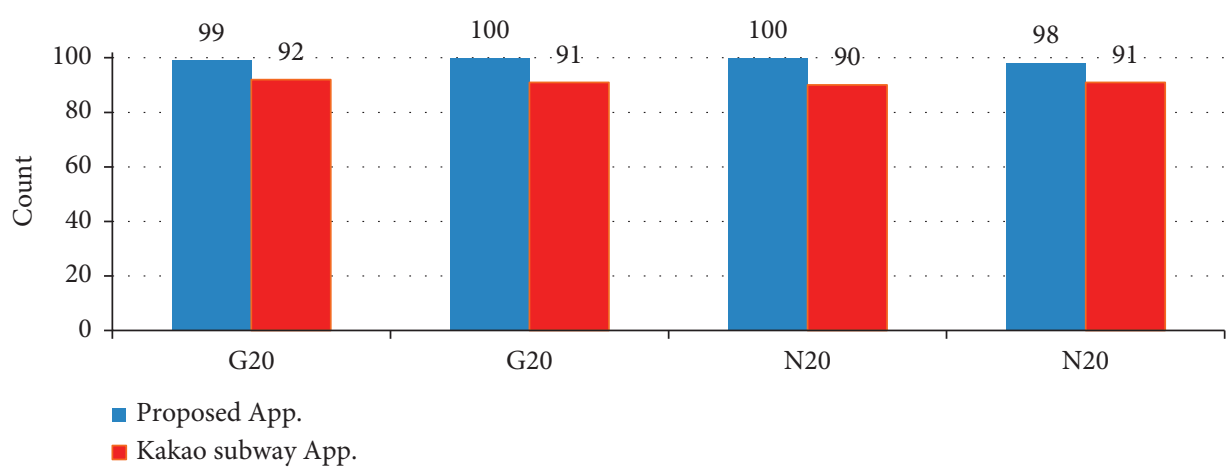

FIGURE 14: Comparison of experiment results with the proposed application and the Kakao subway application.

As the next stage, we tested a comparative experiment with the proposed method and applications using the bus stop Open API. We developed an application using the "Bus Location Information Inquiry" API on a public data portal (https://data.go.kr) used by existing applications. The experiment then used the same number of people and the same devices as in the previous experiment. To avoid overlapping devices, each team was divided into two teams of five and took the same bus. One team used the existing application using bus stop Open API, and the other team used the proposed application to carry out the experiment. The experiment was conducted 100 times for 5 days, and the results of the disembarking notification experiment are shown in Figure 17.

In Figure 17, the proposed method delivered notifications with $100 \%, 99 \%, 98 \%, 100 \%$, and $100 \%$ accuracy, with an average accuracy of $99.4 \%$. We think that 99 on iPhone 11 and 98 on iPhone 12 did not deliver the disembarking notification because participants were either at the farthest distance from the speaker or because participants blocked the microphones of their smart devices. On the other hand, the existing application shows $96,94,95,97$, and 94 accurate notifications, with an average accuracy of $95.2 \%$. In most cases, $100 \%$ accuracy is not seen. We think that the reason is that when continuously transferring GPS values of the bus to the server, processing the data, and providing it to the Open API, the data are sometimes missing, and the current bus's location value is delivered after the stop.

As a final experiment, we measured the battery consumption of each smart device at the beginning of the above comparative experiment. Prior to the start of the experiment, the battery in each smart device started at 100\% charge, and battery checking was done four times at the end station in each direction. The battery consumption experiments refrained from using other applications, just as in the experiments on subways. Figure 18 shows the result of the battery consumption of the proposed methods and the existing application using the Open API. 


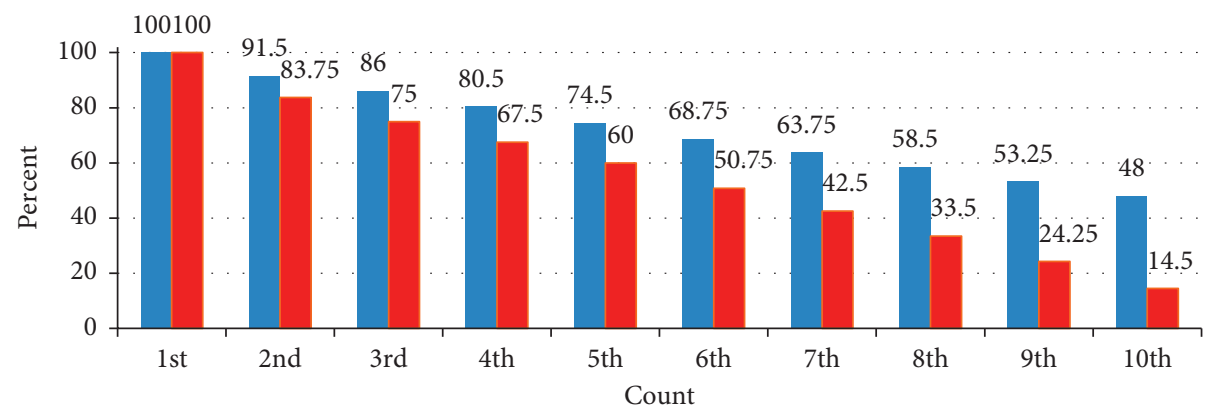

- Proposed App.

- Kakao subway App.

FIGURE 15: Results of the comparison experiment about battery consumption on subways.

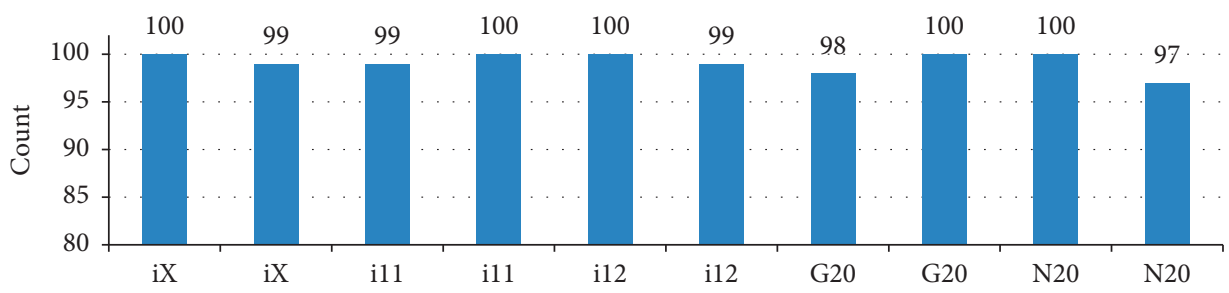

FIGURE 16: The results on the city bus of the paired high frequencies from each smart device.

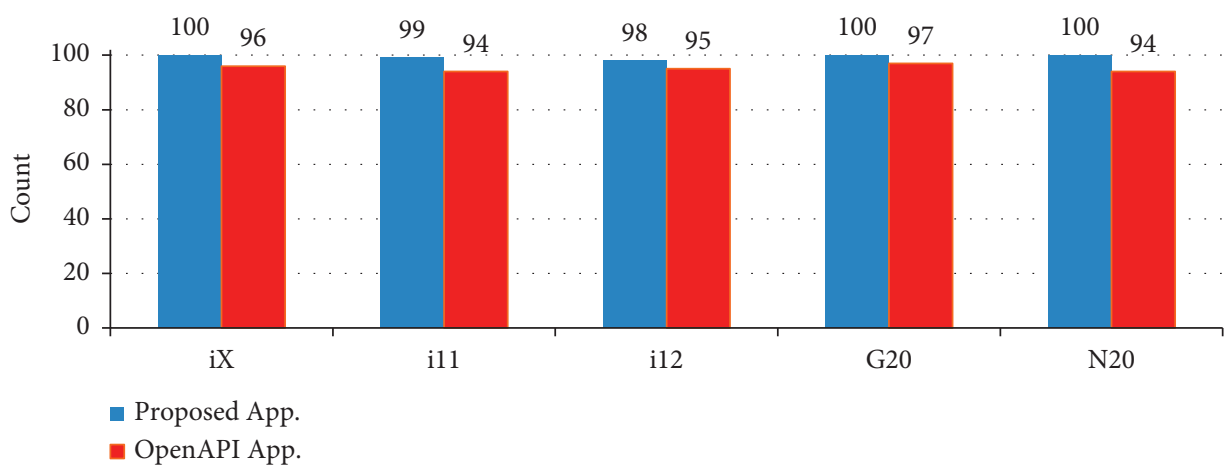

FIgURE 17: Comparison experiment results for the proposed application and Open API application.

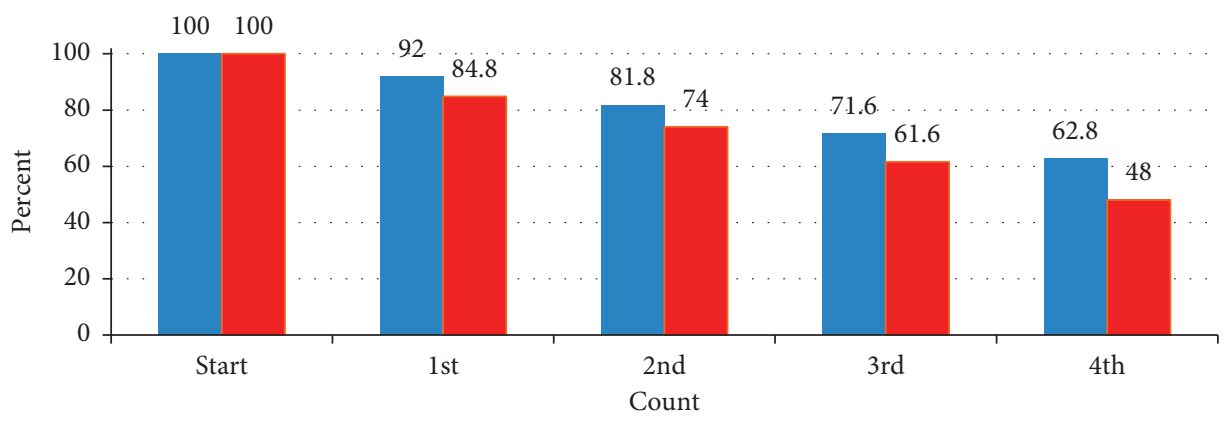

- Proposed App.

- OpenAPI App.

FigURE 18: Results of the comparison experiment about battery consumption on city buses.

As shown in Figure 18, devices with the proposed applications were reduced by $8 \%, 18.2 \%, 28.4 \%$, and $37.2 \%$, resulting in a total of $37.2 \%$ battery consumption during the day's experiment. On the other hand, the existing applications using Open API were reduced by $15.2 \%, 26 \%, 38.4 \%$, and $52 \%$, resulting in a total of $52 \%$ battery consumption. 
We expect that the reason why the existing application consumed more than $14 \%$ of battery compared to the proposed applications is that the existing application uses LTE to continuously import data from servers provided by public data portals, processing the imported data and processing this part. Therefore, as shown by experiments on subways and city buses, the disembarking notification application using high-frequency signals proposed in this paper will be a useful technique for users using public transportation to get off without missing a destination, even if they are enjoying different content.

\section{Conclusions and Future Research}

In this paper, we proposed an application and server system that provides disembarking notifications to users in a new way using built-in microphones of smart devices and speakers installed in public transportation. Through various experiments, it was confirmed that the proposed application shows the value of the next arrival station from a high-frequency signal with station guidance when using the subway and the public city bus. In addition, when the station set by the user is reached, it was confirmed that the application performed accurately for the users by delivering a disembarking notification. Thus, the proposed service system will be a useful service for providing passengers using public transportation, such as subways, buses, and so on, with accurate disembarkation notifications as long as the user sets the destination, even if they are concentrating on enjoying other content. Moreover, it could be an excellent technology that can be commercialized in various public transportation services around the world to announce the next destination through speakers.

However, the proposed application has problem of being very specific to public transportation services that announce getting-off stations through built-in speakers. Therefore, as a further study, even if there is no guiding broadcast for getting off, we will try to achieve $100 \%$ bus and subway disembarking notification accuracy by studying new algorithms and service systems that can generate high-frequency signals by combining GPS data analysis and Wi-Fi fingerprint technology. Finally, in this paper, we use inaudible frequencies in the audible frequency range so that the accuracy of signal transmission by various surrounding environments and noises can be affected. Therefore, we will conduct further experiments on improving the accuracy of data transmission for inaudible frequencies.

\section{Data Availability}

No data were used to support this study.

\section{Conflicts of Interest}

The author declares that there are no conflicts of interest.

\section{Acknowledgments}

This research was supported in part by Ministry of Education, under Basic Science Research Program (NRF2020R1F1A1048133).

\section{References}

[1] H. Kasahara, M. Mori, M. Mukunoki, and M. Minoh, "Transportation mode annotation of tourist GPS trajectories under environmental constraints," in Proceedings of the Information and Communication Technologies in Tourism 2015, pp. 523-535, Lugano, Switzerland, February 2015.

[2] J.-H. Park, S.-H. Kang, and Y.-G. Seo, "Advanced bus information system using smart phone GPS," Journal of the Korea Society of Computer and Information, vol. 19, no. 12, pp. 247-255, 2014.

[3] H.-H. Jung, C.-S. Nam, and D.-R. Shin, "A subway arrival notification system using iBeacon," Journal of KIISE, vol. 42, no. 2, pp. 272-279, 2015.

[4] J. Y. Lee, Y. S. Ryu, and J. H. Hwang, "Development of guidance app for public transportation," Journal of Digital Contents Society, vol. 18, no. 1, pp. 115-121, 2017.

[5] H. Choi, J. Hong, and Y. Yoon, "Intelligent personalized transport alert system with edge computing," in Proceedings of the Advances in Computer Science and Ubiquitous Computing, pp. 251-258, Springer, Singapore, January 2021.

[6] M. Chung, "Detection system of robust high frequency range via noise collection and analysis," Journal of The Korea Society of Computer and Information, vol. 22, no. 1, pp. 71-76, 2017.

[7] S. Oebb, https://www.oebb.at/de/fahrplan/fahrplanauskunft/ scottymobil, 2014.

[8] R. Koutny and K. Miesenberger, "PONS-Mobility assistance on footpaths for public transportation," in Proceedings of the 13th European Conference on the Advancement of Assistive Technology, pp. 440-446, Budapest, Hungary, January. 2015.

[9] R. Koutny, P. Heumader, and K. Miesenberger, "A mobile guidance platform for public transportation," in Proceedings of the International Conference on Computers for Handicapped Persons, pp. 58-64, Springer, Cham, Paris, France, July 2014.

[10] M. A. Ayob, M. N. F. Saaid, K. M. Dimyati, and K. A Maarof, "Destination alarm notification for public transportation passenger using Geo-Fence in mobile App," in Proceedings of the IOP Conference Series: Earth and Environmental Science, vol. 169, no. 1, Article ID 012087, Kuala Lumpur, Malaysia, April. 2018.

[11] B. Y. O. Low, S. H. Dahlan, and M. H. Abd Wahab, "Real-time bus location and arrival information system," in Proceedings of the 2016 IEEE Conference on Wireless Sensors (ICWiSE), pp. 50-53, Langkawi, Malaysia, October. 2016.

[12] M. You and E. Rhee, "Smart bus system using BLE beacon and computer vision," Journal of Institute of Korean Electrical and Electronics Engineers, vol. 22, no. 2, pp. 250-257, 2018.

[13] P. Bihler, P. Imhoff, and A. B. Cremers, "SmartGuide - a smartphone museum guide with ultrasound control," Procedia Computer Science, vol. 5, pp. 586-592, 2011.

[14] M. Chung and I. Ko, "Data-sharing method for multi-smart devices at close range," Mobile Information Systems, vol. 2015, Article ID 931765, 11 pages, 2015.

[15] M. Chung, "Effective near advertisement transmission method for smart-devices using inaudible high-frequencies," Multimedia Tools and Applications, vol. 75, no. 10, pp. 58715886, 2015.

[16] M. Chung, "Short distance data transmission method using inaudible high-frequencies between smart devices," Telecommunication Systems, vol. 70, no. 4, pp. 583-594, 2018.

[17] J. B. Kim, J. E. Song, and M. K. Lee, "Authentication of a smart phone user using audio frequency analysis," Journal of Korea Institute of Information Security and Cryptology, vol. 22, no. 2, pp. 327-336, 2012. 